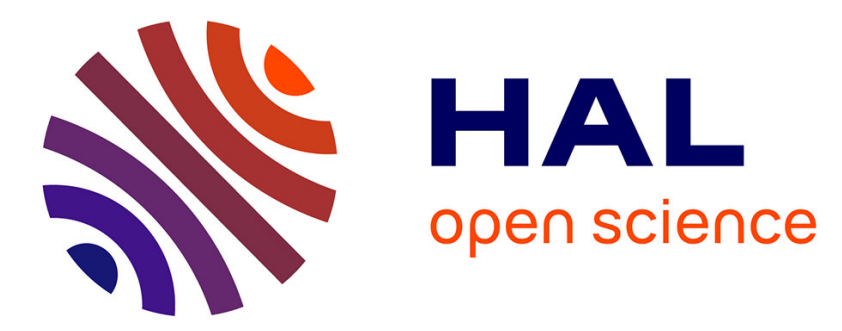

\title{
Quality Control Initiative on the Evaluation of the Dysmegakaryopoiesis in Myeloid Neoplasms: Difficulties in the Assessment of Dysplasia
}

Jean E. Goasguen, John M. Bennett, Barbara J. Bain, Richard D. Brunning, Maria-Teresa Vallespí, Masao Tomonaga, Gina Zini, Alain Renault

\section{To cite this version:}

Jean E. Goasguen, John M. Bennett, Barbara J. Bain, Richard D. Brunning, Maria-Teresa Vallespí, et al.. Quality Control Initiative on the Evaluation of the Dysmegakaryopoiesis in Myeloid Neoplasms: Difficulties in the Assessment of Dysplasia. Leukemia Research, 2016, 45, pp.75-81. 10.1016/j.leukres.2016.04.009 . hal-01301508

\section{HAL Id: hal-01301508 \\ https://hal-univ-rennes1.archives-ouvertes.fr/hal-01301508}

Submitted on 12 Sep 2016

HAL is a multi-disciplinary open access archive for the deposit and dissemination of scientific research documents, whether they are published or not. The documents may come from teaching and research institutions in France or abroad, or from public or private research centers.
L'archive ouverte pluridisciplinaire HAL, est destinée au dépôt et à la diffusion de documents scientifiques de niveau recherche, publiés ou non, émanant des établissements d'enseignement et de recherche français ou étrangers, des laboratoires publics ou privés. 


\section{Highlights :}

1. The presence of micromegakaryocytes correlates strongly with myeloid neoplasia

2. Micromegakaryocytes can be identified reliably

3. Multinucleated megakaryocytes are not specific for myeloid neoplasia

4. Cytoplasmic dysplasia is not useful in recognising MDS

5. 'Possibly dysplastic' cells should be excluded from counts in suspected MDS 


\section{Quality Control Initiative on the Evaluation of the Dysmegakaryopoiesis in Myeloid Neoplasms: Difficulties in the Assessment of Dysplasia}

Jean E. Goasguen ${ }^{1}$, John M. Bennett ${ }^{2}$, Barbara J. Bain ${ }^{3}$, Richard D. Brunning ${ }^{4}$, Maria-Teresa Vallespí ${ }^{5}$, Masao Tomonaga ${ }^{6}$, Gina Zini $^{7}$, and Alain Renault ${ }^{8}$ : The International Working Group on Morphology of MDS (IWGM-MDS).

1-University of Rennes (France), 2-University of Rochester (NY, USA), 3-Imperial College (London, UK), 4University of Minnesota (USA), 5-Vall d’Hebrón Hospital (Barcelona, Spain), 6-University of Nagasaki (Japan), 7-Haematology Institute, Catholic University of Sacred Heart, (Rome, Italy), 8-University of Rennes and INSERM CIC1414, France

Correspondence to:

Jean E. Goasguen MD, PhD

University of Rennes - France

Consultant in Hematology

Personnal address:

4 quai Emile Zola - 35000 RENNES France

Jean.goasguen@orange.fr

$+33(0) 299792404$

Key words: Dysmegakaryocytopoiesis, Dysmegakaryopoiesis, Myelodysplastic syndrome, Dysplastic features, Dysmyelopoiesis, Megakaryocytes.

\section{SUMMARY}

Evaluation of megakaryocyte morphology is difficult but can be essential for the diagnosis of myelodysplastic syndromes (MDS) and other myeloid neoplasms. We agreed upon descriptions and provided images of megakaryoblasts and of normal and dysplastic megakaryocytes, which were used as a basis for assessing the concordance of expert morphologists in their recognition. We showed a high rate of concordance for the recognition of micromegakaryocytes and confirmed their strong association with hematologic neoplasia, including MDS. Concordance was also found to be good for the recognition of multinucleated megakaryocytes, which showed a significant association with MDS. However cytoplasmic abnormalities were found not to be useful in MDS recognition. The occurrence of appreciable numbers of nonlobulated and hypolobulated megakaryocytes in individuals without a myeloid neoplasm was confirmed. We demonstrated that subjects without a myeloid neoplasm can have some megakaryocytes that are assessed as 'dysplastic' or 'possibly dysplastic' and that to avoid 
over diagnosis of dysplasia, 'possibly dysplastic' forms should be excluded from the count of dysplastic cells. Our results demonstrate that the nature as well as the presence of megakaryocyte dysplasia is important in the diagnosis of MDS; although evaluation of 30 megakaryocytes is strongly recommended, it may be possible to recognize diagnostically important dysplasia when fewer megakaryocytes are present but highly diagnostic forms are seen. 


\section{INTRODUCTION}

The diagnosis of low grade myelodysplastic syndromes is greatly dependent on morphologic evaluation of blood and bone marrow films for the detection of dysplasia in myeloid cells. The appearance of micromegakaryocytes in the peripheral blood, associated with atypical forms of megakaryocytes in the bone marrow has been noted in the literature from more than hundred years (1), but it was only in 1982 that most of the abnormalities were described under the designation 'Dysplastic features' by the French-American-British (FAB) group (2). These observations were then complemented in 1985 by the recognition of the cytologic features of megakaryoblasts (3). More recently in 2008 the World Health Organization (WHO) expert group reiterated the features of megakaryoblasts and dysplastic megakaryocytes, including micromegakaryocytes and megakaryocytes with hypolobulated or nonlobulated nuclei (4).

The 2008 WHO Classification of Tumours of Haematopoietic and Lymphoid Tissues requires the recognition of dysplastic features in the diagnosis of the myelodysplastic syndromes (MDS) and acute myeloid leukemia with myelodysplasia-related changes (AML-MRC) $(4,5)$. In the case of MDS, the presence of $10 \%$ or more dysplastic cells restricted to one myeloid lineage is required for a diagnosis of refractory cytopenia with unilineage dysplasia - MDS with single lineage dysplasia (MDS-SLD) (4-6) while the diagnosis of refractory cytopenia with multilineage dysplasia - MDS with multilineage dysplasia - (6) requires the presence of dysplasia in at least $10 \%$ of cells in two or more myeloid lineages. Recognition of AML-MRC on the basis of morphologic abnormalities requires at least $50 \%$ of cells in at least two lineages to be dysplastic. In the case of the megakaryocyte lineage, examination of a minimum of 30 cells is required. In many cases both the degree and characteristics of the dysplasia readily lead to the diagnosis of MDS or AML-MRC. In other instances, both the percentages of dysplastic cells and the characteristics of the dysplasia are marginal, resulting in uncertainty with regard to both the diagnosis and the classification. This is particularly so for cells of the megakaryocytic lineage where less is known of the maturation sequence and morphologic features in normal marrow. Well recognized dysplastic forms include micromegakaryocytes and nonlobulated megakaryocytes of normal size. The former have long been recognized as almost pathognomonic of MDS (7) while the latter show an abnormality that is less specific for a hematologic neoplasm but is highly characteristic of MDS with an isolated deletion of $5 q$ (the ' $5 q$ - syndrome'). There are other forms, such as multinucleated megakaryocytes, that are less strongly linked to MDS since they are occasionally seen in non-neoplastic disorders and even in the bone marrow of healthy subjects (8).

Even for experienced morphologists the decision as to whether a megakaryocyte is a normal variant or dysplastic can be difficult. For this reason, the International Working Group on Morphology of Myelodysplastic Syndromes (IWGM-MDS) set out to provide a comprehensive description of normal and dysplastic megakaryocytes and to assess the concordance between hematologists and hematopathologists in the recognition of these forms on the basis of the descriptions and illustrations provided. The correlation of specific individual features with diagnosis was also evaluated.

\section{MATERIALS AND METHODS}


A planning group of the IWGM-MDS (JEG, RDB, BJB, JMB) met in London in October 2014 to examine blood films and bone marrow aspirates using a multi-headed microscope. In addition, electronic images were analyzed collectively at the meeting and also individually before and after the meeting. A table of morphologically distinguishable subtypes of megakaryocyte was prepared (Table 1). Descriptions of normal and abnormal megakaryocytes were agreed on and appropriate illustrative images were selected to serve as an introductory tutorial.

\section{Descriptions of the morphologic categories}

1- A megakaryoblast is a normal cell but is not recognized in normal bone marrow aspirates because of its infrequency and the lack of distinctive distinguishing features. When megakaryoblasts are present in increased numbers their nature can be identified by immunophenotyping and their cytologic features can then be defined. Megakaryoblasts are similar in size to myeloblasts. They have a delicate or diffuse chromatin pattern and may have nucleoli. Sometimes they have no distinguishing features (Figure 1: 1a) but in other patients they are observed to have cytoplasmic blebs (Figure 1: 1b) and early platelet-type granulation of the cytoplasm $(9,10)$. They do not produce platelets. Megakaryoblasts are observed in significant numbers in acute megakaryoblastic leukemia (e.g. in acute megakaryoblastic leukemia associated with $\mathrm{t}(1 ; 22)(\mathrm{p} 13.3 ; \mathrm{q} 13.1)$ or in children with Down syndrome, in transient abnormal myelopoiesis (TAM) in neonates with Down syndrome, and in megakaryoblastic transformation of myeloproliferative neoplasms (11).

2- An immature megakaryocyte (Figure 1:2) is a normal cell with low ploidy. The nuclear cytoplasmic ratio is high. The nucleus shows some chromatin condensation and may show early lobulation. The cytoplasm is scanty and basophilic, often forming blebs but with few if any granules.

3- A normal mature megakaryocyte (Figure 1: 3 ) is a large polyploid cell, ranging from $4 \mathrm{~N}$ (tetraploid) to $64 \mathrm{~N}$. Its nucleocytoplasmic ratio is lower than that of the immature megakaryocyte and its cytoplasm is less basophilic. Sometimes the cytoplasm is demarcated into a central basophilic zone and a peripheral more weakly basophilic zone. With increasing cytoplasmic maturation, there is granule formation and sometimes demarcation of proplatelets is apparent within the cytoplasm. Platelets may be apparent being detached from the surface. The nucleus shows chromatin condensation and is usually lobulated but it should be noted that some normal megakaryocytes are large but with nonlobulated nuclei and are separately identified (see below).

4- A normal late megakaryocyte (Figure 1: 4) has shed almost all its cytoplasm as platelets. All that remains is a thin rim of weakly basophilic cytoplasm. These cells are often referred to as 'bare' or 'naked' nuclei but careful inspection shows a thin rim of residual cytoplasm. The nucleus shows pronounced chromatin condensation and may be hyperchromatic since it is near to apoptosis.

5- A megakaryocyte with a nonlobulated nucleus and a normal size (Figure 1:5) is a cell of normal size with a nucleus that is round or slightly oval. The cytoplasm is usually mature. Such cells can be seen in small numbers in the bone marrow of healthy individuals but they are increased in 
number in some patients with MDS and are particularly characteristic of the $5 q$ - syndrome. It should be noted that the term 'mononuclear megakaryocyte' should not be used when what is intended is a nonlobulated megakaryocyte. The majority of megakaryocytes are mononuclear so the term should only be used when it is necessary to make a distinction from a binucleated or other multinucleated megakaryocyte.

6- A megakaryocyte with a hypolobulated nucleus and a normal size (Figure 1: 6) is a cell of normal size with a nucleus that is less lobulated than expected for the size of the nucleus. Such cells can be seen in normal bone marrow but numbers are increased in some patients with MDS, including the $5 q$ - syndrome.

7- A small megakaryocyte with a hypolobulated or nonlobulated nucleus (Figure 1: 7) is a cell that is smaller than average with a nucleus that is either less lobulated than average or is nonlobulated. Such cells are part of the spectrum of normal mature megakaryocytes and should only be commented on and classified separately when they form an increased proportion of megakaryocytes. They are seen in normal bone marrow, in MDS, and in chronic myeloid leukaemia $(\mathrm{CML})$, the latter condition being characterized by a reduction in size and ploidy of megakaryocytes (12).

8- A micromegakaryocyte is usually a mononuclear diploid cell, with a nucleus similar in size to that of a myeloblast or promyelocyte with the cell being less than $30 \mu \mathrm{m}$ in diameter (Figure 1: $8 a$ and $8 b$ ). Some micromegakaryocytes are binucleated tetraploid cells (Figure 8a); they can be up to twice the size of a mononuclear micromegakaryocyte but often they are not much larger. Micromegakaryocytes may be immature with scanty basophilic cytoplasm or mature (Figure 1: $8 b)$ with more plentiful cytoplasm that contains many granules. Sometimes they are in the process of shedding their cytoplasm as platelets so they appear as small hyperchromatic nuclei with strands of cytoplasm fragmenting into platelets. Micromegakaryocytes are characteristic of MDS. They can also be seen in TAM, in myelodysplastic/myeloproliferative neoplasms (MDS/MPN), and in the accelerated phase or transformation of myeloproliferative neoplasms (MPN). In the accelerated phase or transformation of CML there may be sheets of micromegakaryocytes with small, lymphocyte-sized nuclei and plentiful cytoplasm fragmenting into platelets.

9- A multinucleated megakaryocyte (Figure 1: 9) is a cell of normal size with two or more nuclei. Binucleated micromegakaryocytes should be categorized as such rather than as multinucleated megakaryocytes since the restricted size is of more diagnostic significance than the presence of two nuclei. Multinucleated megakaryocytes are seen in small numbers in normal bone marrow aspirates but in larger numbers in some patients with MDS.

10- A large megakaryocyte with a hyperlobulated nucleus (Figure 1: 10) is a cell that is larger than average size and has more nuclear lobulation than average. Some such megakaryocytes are seen in normal bone marrows but when they are present as an increased proportion they should be commented on. Increased numbers are seen sometimes in MDS but they are more frequent in MDS/MPN and in MPN, particularly essential thrombocythemia.

11- Megakaryocytes with cytoplasmic abnormalities may have cytoplasmic vacuolation (Figure 1: 11a), cytoplasmic hypogranularity or agranularity (Figure 1: 11b) or persisting basophilia so that there is nucleocytoplasmic asynchrony (Figure 1: 11c). 
12- A damaged megakaryocyte is one with disruption of the cytoplasmic membrane as a result of the spreading of the film. Often there is also disruption of the nucleus. A damaged megakaryocyte, not assessable, has been so damaged by crushing that it is not possible to determine whether it was initially cytologically normal or not. It is important to recognize damaged (crushed, squashed, flattened) megakaryocytes so that they are not confused with dysplastic forms. A frequent effect of damage during spreading of the film is that the nucleus is fragmented; such cells should not be confused with multinucleated megakaryocytes or other dysplastic forms. A cell classified as 'damaged megakaryocyte, not assessable' should be disregarded when assessing the proportion of dysplastic forms.

13- This item is reserved for a cell that is considered not to be from the megakaryocytic lineage but was included among some of the images. Therefore no photograph is provided.

\section{Morphologic analysis}

Fourteen cases were selected to evaluate concordance of the group including five MDS, one CML, one CML in blast crisis, one TAM, and six non-neoplastic cases designated 'non-MDS' (three autoimmune thrombocytopenia (ITP), one healthy adult control, and two acute lymphoblastic leukemia (ALL) in remission without any maintenance therapy). The five MDS cases were two MDS-SLD and three myelodysplastic syndrome with excess blasts (6). The MDS, CML, and TAM cases were included because the presence of dysmegakaryopoiesis had already been recognized.

All megakaryocytes from each bone marrow film were photographed, explaining the variable number of pictures (cells) for each case. All images had the same definition (Leica microscope with $x 63$ objective under oil). The seven members of the IWGM-MDS group received these pictures without knowledge of the diagnosis or classification. They had to assign each cell to one of the 13 designated categories and decide if the cell was 'Dysplastic' (1) or not (0) or 'possibly dysplastic' (2). For each case an overall judgment as to whether or not the case showed dysplasia was required.

\section{Statistics}

All data were centralized and statistical analysis was performed using SAS software. Two methods were used: 1- For each cell (7 evaluations/cell) the final category was decided by using the "majority" (at least 4/7) and consequently we have the final subtype (1 to 13) with the degree of agreement for every cell; 2- For each case (number of cells per case $x 7$ evaluations) it was therefore possible to submit 'independently' all data directly for statistical analysis.

\section{RESULTS}

A total of 387 cells from the 14 selected cases were evaluated independently by seven morphologists. 2700 evaluations were collected (9 missing). Since 46 cells were difficult to classify (agreement <4/7) they were submitted a second time (again with blinding as to the diagnosis) before statistical analysis. 39 cells of these 46 had been assigned to more than one category with only $32 \%$ agreement. They were reclassified with $55 \%$ agreement, 32 being assigned to only one category. 
Among the 387 cells, eight were evaluated as ' 13 ' (not megakaryocyte lineage) and were excluded. Finally, 379 megakaryocytes were classified and 94.7 percent (359 cells out of 379) of these were assigned to only one category; 18 cells were assigned to two categories (for example categories ' 2 ' and ' 3 ' for 4 cells) and 2 cells were assigned to 3 categories (see Table 2). Agreement for these identifications was: $7 / 7$ for 46 cells, $6 / 7$ for 66 cells, 5/7 for 91 cells, $4 / 7$ for 103 cells, $3 / 7$ for 52 cells, and $2 / 7$ for one cell, which is $69.36 \%$ agreement for all cells. Among these 359 cells $83.9 \%$ were classified with at least 4/7 agreement (majority) between morphologists. The decision as to the category was performed with mean agreement of $77 \%$ and $66 \%$ for the non-MDS and MDS groups respectively.

All cases that were included as having dysmegakaryopoiesis were confirmed, and non-dysplastic cases were similarly confirmed as such. When the panel members had to determine if an individual cell was dysplastic or not, usually only a few cells were considered to be dysplastic in the non-MDS group - far from enough cells for the case to be considered dysplastic.

Normal megakaryocytes (category 3 ) comprised $69.3 \%$ and $19.7 \%$ in the non-MDS and MDS groups respectively $(p<0.0001)$.

Since micromegakaryocytes were well represented in this series and are viewed as one of the hallmarks of megakaryocytic dysplasia we examined both subtypes $8 \mathrm{a}$ and $8 \mathrm{~b}$ for concordance. The results demonstrated a high degree of concordance for both $8 \mathrm{a}$ (42/45) and 8b (33/36). Minor disagreements were noted between micromegakaryocytes and cells that were called: 'normal immature' and 'small nonlobulated' (see Table 2). Micromegakaryocytes showed a strong association with MDS and were prominent in the baby with TAM; in MDS 47/142 cells (33.1\%) were unanimously identified as micromegakaryocytes and in the case of TAM, $26 / 43$ cells $(60.5 \%)$. Significant numbers of micromegakaryocytes were seen in the patient with CML (7/31 cells, 22.6\%) but not in the non-MDS group.

For Category 9 (multinucleated megakaryocyte with two or more separate nuclei), another characteristic feature of megakaryocytic dysplasia well recognized in MDS and AML, the concordance was $21 / 25,84 \%$ (see Table 2). Multinucleated megakaryocytes showed an association with MDS, 19/142 cells assessed (13.4\%), but were also observed occasionally in other myeloid neoplasms ( $2 / 74$ cells assessed $(2.7 \%)(p=0.0253)$ and in non-MDS, 4/171cells assessed $(2.3 \%)(p=0.0002)$.

Normal sized nonlobulated megakaryocytes showed only a weak association with a diagnosis of MDS (7.7\% of cells in comparison with $7 \%$ of cells in non-MDS) but it should be noted that no case of the $5 q-$ syndrome was included in this study.

None of the cytoplasmic abnormalities was found to clearly segregate with MDS or with MDS plus other hematologic neoplasms (Table 2).

To evaluate the dysplastic character of each cell, data may be considered by two methods: 'Majority' or 'Independently". We recorded 2700 responses but 55 evaluations concerned the ' 13 ' category (leaving 2645 data to discuss). Code ' 0 ' (non-dysplastic) was used 1228 times (46.4\%), code ' 1 ' (dysplastic = yes) was recorded 1150 times (43.5\%) and code ' 2 ' (possibly dysplastic) 267 times (10.1\%). The results of 
evaluation by the 'majority' and 'independent' methods are summarized in Table 3 in which MDS and non-MDS cases may be compared. The percentage of dysplastic cells is necessarily increased if 'possibly dysplastic' (code 2) is added to 'dysplastic yes' (code 1). This point has an important impact for the 'nonMDS' group, leading to an increase from $3.0 \%$ dysplastic cells to $14.5 \%$ and from $11.1 \%$ to $22.7 \%$ by the majority and independent methods respectively.

Results for the 'non-MDS' group are shown separately in Table 4. The same increase is observed in the three cases of ITP, the two ALL cases, and the healthy adult control (ML), with ML increasing from $5.6 \%$ to $22.2 \%$ with the 'majority' method and from $18.3 \%$ to $35.3 \%$ with the 'independent' method. This point is discussed below.

\section{DISCUSSION}

It is noteworthy that the recognition of the different features of the megakaryocytic lineage can be difficult. Descriptions varied somewhat between the FAB and WHO publications and even experienced morphologists may have difficulty agreeing on a categorization. Some dysplastic features are already well described in the literature, including the features of micromegakaryocytes and multinucleated megakaryocytes and the particular characteristics that are the hallmark of MDS with isolated $5 q$ deletion. However, even for these fairly well defined types, there is room for differences of opinion. For example, a micromegakaryocyte may be mature or immature and the latter may be confused with an immature precursor or a megakaryoblast. Similarly, a megakaryocyte nucleus may have very slight lobulation and thus might be classified as either nonlobulated or hypolobulated.

For these reasons we decided to carry out a quality control exercise to determine the reproducibility of the observation of specific dysplastic features by a group of experienced morphologists, by circulating digital images from MDS and non-MDS cases.

The first conclusion was that $94.7 \%$ of megakaryocytes could be assigned to a single category with 69.36\% agreement (4.85/7) even though there were 12 categories, but it must be noted that the morphologists were able to utilize the tutorial photographs during the evaluation and that re-evaluation of cases that lacked consensus was necessary to achieve this degree of agreement. We obtained a similar agreement ( $63 \%$ or $66 \%$ ) for the identification of 'immature' and 'mature' micromegakaryocytes permitting the recognition of $93 \%$ as either $8 \mathrm{a}$ or $8 \mathrm{~b}$. This indicates that the distinction between these two subtypes is possible.

All investigators had to classify each case as dysplastic or not according to the WHO guidelines. All MDS cases (which had already been identified as having dysmegakaryopoiesis in the referring hospital) were confirmed with $7 / 7$ agreement. The non-MDS group was generally considered to be without dysplasia with an agreement of 5.5/7. If we compare these results with the results that are presented in Tables 3 and 4 , it is clear that the addition of the item 'possibly dysplastic' increases the percentage of dysplastic cells in the two groups considerably above $10 \%$ for some of the non-MDS group including two ITP cases (20.3 and $19.5 \%$ respectively). Furthermore in the control subject who was a healthy hospital staff 
member the majority of the group decided that 2 cells out of 36 were dysplastic (5.6\%). In addition to these two cells, a further 6 cells were coded as 'possibly dysplastic'. The addition of 'possibly dysplastic' increases the dysmegakaryopoiesis from $5.6 \%$ to $22.2 \%$. Of the 252 independent evaluations, 46 cells were evaluated as dysplastic (18.3\%) and a further 43 cells as 'possibly dysplastic'; if 'possibly dysplastic' cells are included no less than $35.3 \%$ of cells are regarded as 'dysplastic'.

The degree of variation in morphologic features of megakaryocytes in the bone marrows of healthy volunteers is not widely appreciated, leading to possible over interpretation of normal variation as evidence of dysplasia. In addition to our observations on the healthy control subject, Bain observed 19 of 50 healthy volunteers to have one or two megakaryocytes out of 20 examined that could be interpreted as dysplastic since they were either nonlobulated (14 subjects) or multinucleated (4 subjects); one subject had both a large binucleated megakaryocyte and a large nonlobulated megakaryocyte (8). Despite the proposal by Matsuda et al in 2008 (13) that dysplasia be graded as "highly specific" and "less specific" we therefore recommend that cells considered to be possibly dysplastic' are excluded from the count of dysplastic cells as these not infrequently merely represent normal variation. Accepting only megakaryocytes that are considered to be definitely dysplastic (code 1) in the count may reduce the number of patients with MDS in whom megakaryocyte dysplasia can be recognized but avoids the risk of misclassifying cases as dysplastic when they are not.

Consideration must also be given to how many megakaryocytes need to be examined to decide on the presence or absence of dysmegakaryopoiesis with a reasonable degree of reliability. The WHO classification requires assessment of at least 30 megakaryocytes (4) in bone marrow films or sections, but in a number of cases this is not possible on films from a single bone marrow aspirate. In 2000 Germing et al proposed that dysmegakaryopoiesis be defined by the presence of at least 10 dysplastic megakaryocytes out of 25 cells evaluated (40\%) (14). A count of 25 megakaryocytes with a threshold at $40 \%$ (with prognostic significance) was also proposed in 2007 by Matsuda et al (15) and subsequently by Della Porta et al (16). In our study, the number of megakaryocytes for the MDS group per patient was: 19, 29, 30, 31 and 32. Even when 30 megakaryocytes are evaluated, the confidence limits of a count of $10 \%$ dysplastic cells are very broad and therefore it seems prudent to evaluate 30 megakaryocytes when that number can be found. It may however, be possible to identify dysplasia when fewer than 30 cells are available, depending on the nature of the dysplastic feature. We and others have found the presence of micromegakaryocytes to correlate strongly with hematologic neoplasia whereas we found cytoplasmic abnormalities to show only a weak correlation. Multinucleated megakaryocytes were found to be useful indicators of dysplasia. Although not demonstrated to be useful in the current study, which did not include any examples of MDS with isolated deletion of 5q, megakaryocytes with nonlobulated or hypolobulated nuclei are also clearly important in the recognition of the $5 q$ - syndrome; however the appreciable number that can be observed in patients without a myeloid neoplasm needs to be borne in mind.

In conclusion, we have shown that even experienced morphologists can have difficulty in reaching consensus as to whether or not an individual cell is dysplastic. Recognition of the categories of cell described above is useful for identification or exclusion of dysplasia with consideration also being given 
to the specificity of a given feature for MDS. To avoid over-interpretation of atypical features we recommend that only cells considered to be definitely 'dysplastic' be included in the count. It is unrealistic to hope to evaluate more than 30 megakaryocytes but it must be acknowledged that even with this number of cells being assessed, the confidence limits of a low count of dysplastic cells are wide. Consideration could therefore be given to requiring a higher threshold than $10 \%$ for the recognition of significant megakaryocyte dysplasia. Others have suggested $40 \%$ as a threshold $(14,15,16)$. Consideration of our data suggests a threshold of 20 or $25 \%$ could also be considered.

\section{References}

1. Minot GR: Megakaryocytes in the peripheral circulation. J Exp Med 1922; 36: 1.

2. Bennett JM, Catovsky D, Daniel MT, Flandrin G, Galton D, Gralnick H et al. Proposals for the classification of the myelodysplastic syndromes. Br J Haematol 1982; 51: 189-199.

3. Bennett JM, Catovsky D, Daniel MT, Flandrin G, Galton D, Gralnick H et al. Criteria for the diagnosis of acute leukemia of megakaryocytic lineage (M7): a report of the French-AmericanBritish Cooperative Group. Ann Intern Med 1985; 103: 460-462.

4. Brunning RD, Orazi A, Germing U, le Beau MM, Porwit A, Bauman I et al. Myelodysplastic syndromes/neoplasms, overview, In Swerdlow SH, Campo E, Harris NL, Jaffe ES, Pileri SA, Stein $\mathrm{H}$, Thiele J and Vardiman JW (eds), World Health Organization Classification of Tumours of Haematopoietic and Lymphoid Tissues, IARC Press, Lyon, 2008, pp 88-93.

5. Arber DA, Brunning RD, Orazi A, Bain BJ, Porwit A, Vardiman JW et al. Acute myeloid leukaemia with myelodysplasia-related changes, In Swerdlow SH, Campo E, Harris NL, Jaffe ES, Pileri SA, Stein $\mathrm{H}$, Thiele J and Vardiman JW (eds), World Health Organization Classification of Tumours of Haematopoietic and Lymphoid Tissues, IARC Press, Lyon, 2008, pp 124-126.

6. Arber DA, Hasserjian RP. Reclassifying myelodysplastic syndromes: what's where in the new WHO and why? Hematology 2015; 2015: 294-298.

7. Kuriyama K, Tomonaga M, Matsuo T, Ginnai I, Ichimaru M. Diagnostic significance of detecting pseudo-Pelger-Huët anomalies and micromegakaryocytes in myelodysplastic syndrome. $\mathrm{Br} \mathrm{J}$ Haematol 1986; 63: 665-669.

8. Bain BJ. The bone marrow aspirate of healthy subjects. Br J Haematol 1996; 94: 206-209.

9. Maldonado J E. Dysplastic platelets and circulating megakaryocytes in chronic myeloproliferative diseases. Il ultrastructure of circulating megakaryocytes. Blood 1974; 43: 811-820.

10. Duchayne E, Fenneteau O, Pages MP, Sainty D, Arnoulet C, Dastugue N et al. Acute megakaryoblastic leukaemia: a national clinical and biological study of 53 adult and childhood cases by the Groupe Francais d'Hematologie Cellulaire (GFHC). Leuk Lymphoma 2003; 44: 49-58.

11. Litz CE, Davies S, Brunning RD, Kurk B, Parkin JL, Gajl Peczalska KJ et al. Acute leukemia and transient myeloproliferative disorder associated with Down syndrome: morphologic immunophenotypic and cytogenetic manifestations. Leukemia 1995; 9: 1432-1439.

12. Franzén S, Strenger G, Zajicek J. Microplanimetric studies on megakaryocytes in chronic granulocytic leukaemia and polycythaemia vera. Acta Haematol 1961; 26: 182-193.

13. Matsuda A, Jinnai I, Miyazaki Y, Tomonaga. Proposals for a grading system for diagnostic accuracy of myelodysplastic syndromes. Clin Leuk. 2008;2:102-106. 
14. Germing U, Gattermann N, Aivido M, Hildebrandt B, and Aul C. Two types of acquired sideroblastic anaemia (AISA): a time-tested distinction. Brit J Haematol 2000, 108: 724-728.

15. Matsuda A, Germing U, Jinnai I, Iwanaga M, Misumi M, Kuendgen A et al. Improvement of criteria for refractory cytopenia with multilineage dysplasia according to the WHO classification based on prognostic significance of morphological features in patients with refractory anemia according to the FAB classification. Leukemia 2007; 21: 678-686.

16. Della Porta MG, Travaglino E, Boveri E, Ponzoni M, Malcovati L, Papaemmanuil E et al. on behalf of Rete Ematologica Lombarda (REL) clinical network in 2014. Myelodysplasias: minimal morphological criteria for defining bone marrow dysplasia: a basis for clinical implementation of WHO classification of myelodysplastic syndromes. Leukemia 2015; 29: 66-75. 


\section{ACCEPTED MANUSCRIPT}

Figure 1: Representation of the different categories that were submitted for evaluation: numbers 1-12 have the significance shown in Table 1; $1 \mathrm{a}$ and $1 \mathrm{~b}$ are two different examples for category 1.
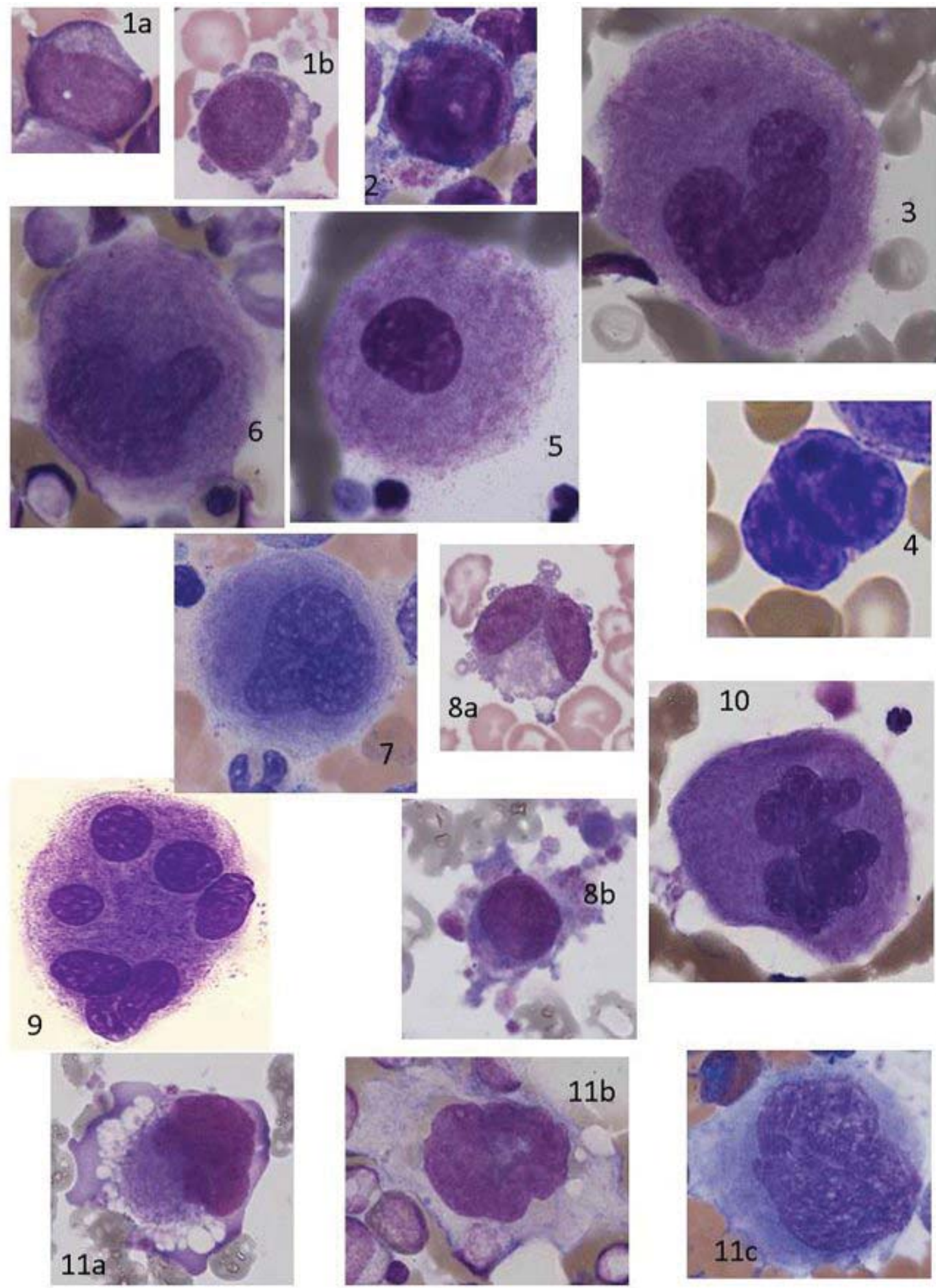
Table 1: Summary of the 13 categories that were used for this evaluation.

1. Megakaryoblast

2. Immature megakaryocyte

3. Normal mature megakaryocyte

4. Normal late megakaryocyte - 'bare nucleus'

5. Megakaryocyte with nonlobulated nucleus, but of normal size

6. Megakaryocyte with hypolobulated nucleus, but of normal size

7. Small megakaryocyte, nonlobulated or hypolobulated

8. Micromegakaryocyte (mononuclear or binucleated)

a. Immature

b. Mature

9. Multinucleated megakaryocyte (two or more separate nuclei)

10. Large megakaryocyte with hyperlobulated nucleus

11. Megakaryocytes with a cytoplasmic abnormality
a. Vacuolation
b. Agranular or hypogranular cytoplasm
c. Persisting basophilia (nucleocytoplasmic asynchrony)

12. Damaged (crushed/squashed) megakaryocyte, not evaluable*

13. Not megakaryocyte lineage

* Damaged megakaryocytes that were assessable were assigned to the relevant category 
Table 2: results of the identification of the 387 images according to the 13 proposed subtypes by 7 morphologists. (Interpretation: first line, 19 cells were assigned to the category ' 1 ' ; line 2 , one cell was interpreted as ' 1 ' by 3 observers and as ' 13 ' also by 3 observers and is therefore classified as $1 / 13$ )

\begin{tabular}{|c|c|c|c|c|c|}
\hline Categories* & CML & TAM & MDS & $\begin{array}{l}\text { Non- } \\
\text { MDS }\end{array}$ & Total \\
\hline 1 & & 16 & 2 & 1 & 19 \\
\hline $1 / 13$ & & & 1 & & 1 \\
\hline 2 & 1 & & 11 & 14 & 26 \\
\hline $2 / 3$ & & & & 4 & 4 \\
\hline $2 / 6$ & & & 1 & & 1 \\
\hline $2 / 7 / 8 a$ & & & 1 & & 1 \\
\hline $2 / 8 a$ & & & 1 & & 1 \\
\hline 3 & 4 & & 26 & 109 & 139 \\
\hline $3 / 10$ & & & & 1 & 1 \\
\hline $3 / 6$ & & & 1 & 3 & 4 \\
\hline 4 & & & & 3 & 3 \\
\hline $4 / 7$ & & & & 1 & 1 \\
\hline 5 & & & 4 & & 4 \\
\hline $5 / 7$ & & & 1 & & 1 \\
\hline 6 & 5 & & 11 & 12 & 28 \\
\hline $6 / 12$ & & & 1 & & 1 \\
\hline 7 & 12 & 1 & 10 & 3 & 26 \\
\hline $7 / 8 a$ & & & & 1 & 1 \\
\hline $7 / 8 a / 8 b$ & & & 1 & & 1 \\
\hline $8 a$ & 2 & 15 & 25 & & 42 \\
\hline $8 a / 8 b$ & 1 & 1 & & & 2 \\
\hline $8 b$ & 4 & 10 & 19 & & 33 \\
\hline 9 & 2 & & 19 & 4 & 25 \\
\hline 10 & & & 2 & 7 & 9 \\
\hline 12 & & & 2 & 3 & 5 \\
\hline 13 & & & 3 & 5 & 8 \\
\hline Total & 31 & 43 & 142 & 171 & 387 \\
\hline
\end{tabular}

* As shown in Figure 1 and Table 1. 
Table 3: Results of the evaluations of the dysplastic character for all patients and one healthy volunteer

\begin{tabular}{|l|l|l|l|l|}
\hline Dysplasia & $\begin{array}{l}\text { Majority for } \\
\text { Dysplasia ('1' only) * }\end{array}$ & $\begin{array}{l}\text { Majority for } \\
\text { Dysplasia ('1'+ '2')* }\end{array}$ & $\begin{array}{l}\text { Independently } \\
\text { Dysplasia ('1' only) }\end{array}$ & $\begin{array}{l}\text { Independently } \\
\text { Dysplasia ('1'+ '2') }\end{array}$ \\
\hline CML (n=2) & $23 / 31(74.2)$ & $26 / 31(83.9)$ & $146 / 217(67.3)$ & $146+30 / 217(81.1)$ \\
\hline TAM (n=1) & $35 / 43(81.4)$ & $43 / 43(100)$ & $239 / 300(79.7)$ & $239+23 / 300(87.3)$ \\
\hline MDS (n=5) & $92 / 139(66.2)$ & $105 / 139(75.5)$ & $636 / 970(65.6)$ & $636+80 / 970(73.8)$ \\
\hline Non-MDS (n=6) & $5 / 166(3.0)$ & $24 / 166(14.5)$ & $129 / 1158(11.1)$ & $129+134 / 1158(22.7)$ \\
\hline Total (n=14) & $155 / 379(40.9)$ & $198 / 379(52.2)$ & $1150 / 2645(43.5)$ & $1417 / 2645(53.6)$ \\
\hline
\end{tabular}

* ' 1 ' = dysplastic; '2' = 'possibly dysplastic' 
Table 4: Details of the evaluation of the "Dysplastic" character by two methods, for the 'non-MDS' group only.

\begin{tabular}{|c|c|c|c|c|}
\hline Diagnosis* & $\begin{array}{l}\text { Majority for } \\
\text { Dysplasia (' } 1 \text { ' only) }+\end{array}$ & $\begin{array}{l}\text { Majority for } \\
\text { Dysplasia }\left({ }^{\prime} 1 \text { '+ '2') }+\right.\end{array}$ & $\begin{array}{l}\text { Independently } \\
\text { Dysplasia (' } 1 \text { ' only) }\end{array}$ & $\begin{array}{l}\text { Independently } \\
\text { Dysplasia ('1' + '2') }\end{array}$ \\
\hline A1 & $1 / 30(3.3)$ & $2 / 30(6.7)$ & $11 / 203(5.4)$ & $11+15 / 203(\mathbf{1 2 . 8})$ \\
\hline $\mathbf{A 2}$ & $1 / 32(3.1)$ & $7 / 32(21.9)$ & $21 / 231(9.1)$ & $21+26 / 231(20.3)$ \\
\hline A3 & $0 / 11(0)$ & 2/11 (18.2) & $5 / 77(6.5)$ & $5+10 / 77 \quad(19.5)$ \\
\hline C1 & $0 / 41(0)$ & $4 / 41(9.8)$ & $34 / 283$ (12.0) & $34+32 / 283(23.3)$ \\
\hline C2 & $1 / 16(6.3)$ & $1 / 16(6.3)$ & $12 / 112(\mathbf{1 0 . 7 )}$ & $12+8 / 112(17.9)$ \\
\hline ML & $2 / 36(5.6)$ & $8 / 36(22.2)$ & $46 / 252$ (18.3) & $46+43 / 252(35.3)$ \\
\hline Total & $5 / 166(3.0)$ & $24 / 166$ (14.5) & $129 / 1158$ (11.1) & $263 / 1158$ (22.7) \\
\hline
\end{tabular}

* $A 1, A 2$, and $A 3$ are autoimmune thrombocytopenia; $C 1$ and $C 2$ are acute lymphoblastic leukemia in remission; $\mathrm{ML}$, is a healthy volunteer.

†' 1 ' = dysplastic; '2' = 'possibly dysplastic' 